\title{
Nobel Lecture: The dilemma of attribution*
}

\author{
David Politzer \\ Department of Physics, California Institute of Technology, Pasadena, California 91125, \\ USA
}

(Published 7 September 2005)

I suspected that there were some members of the live audience who were somewhat apprehensive about sitting through the morning's physics lectures. After all, there were three guys there to talk about one minus sign. If it were just two people and a plus sign, + , one could talk about the $\mid$ and the other about the - - However, to my mind, this year's awards represent or symbolize not just a minus sign but a large body of significant advances in our understanding of fundamental physics and are the work of not just three people but a great many scientists, stretching out over many years and many countries. This is really a prize for that whole community.

Sidney Coleman, my beloved teacher from graduate school, referred to this community as $i$ fratelli fisici, by which he meant the brotherhood of physicists. Most of us spoke at least a bit of broken Italian, a legacy of the grand and highly influential summer schools organized by Nino Zichichi in Erice, Sicily. Indeed, one of my fondest reflections on my particle physics career is having been able to arrive at a train station, virtually anywhere in the world, and be greeted by a total stranger who immediately treated me like an old friend.

I'd love to tell you all their stories, but I certainly don't know them all, nor do I have time (or space) even for those that I do. So I've chosen a few of the people and a few of the stories with which to make a particular point. You can judge for yourself at the end how well I've succeeded. And I'll deal mostly with theorists because I know them best-although I must say that I do regard theoretical physics as a fundamentally parasitic profession, living off the labors of the real physicists.

I'd like to address one particular aspect of the impact of these prizes. To a considerable extent they have come to represent milestones in the progress of science. And it is a testament to the care and wisdom exercised in the selection process just how important the prizes have become. To the public, they spark continued interest in science's most important advances. But even within the world of the scientific experts, the prizes likewise serve as markers of this progress. The use of history in science education may be a contributing factor to why this is so and how it works. As teachers of the next generation of scientists, we always seek to compress and simplify all

\footnotetext{
*The 2004 Nobel Prize in Physics was shared by David J. Gross, David Politzer, and Frank Wilczek. This lecture is the text of Professor Politzer's address in conjunction with the award.
}

the developments that have come before. We want to bring our students as quickly as possible to the frontier of current understanding. From this perspective, the actual history, which involves many variants and many missteps, is a only a hindrance. And the neat, linear progress, as outlined by the sequence of gleaming gems recognized by Nobel prizes, is a useful fiction. But a fiction it is. The truth is often far more complicated. Of course, there are the oft-told priority disputes, bickering over who is responsible for some particular idea. But those questions are not only often unresolvable, they are often rather meaningless. Genuinely independent discovery is not only possible, it occurs all the time. Sometimes a yet harder problem in the prize selection process is to identify what is the essential or most important idea in some particular, broader context. So it's not just a question of who did it, i.e., who is responsible for the work, but what "it" is. I.e., what is the significant "it" that should stand as a symbol for a particularly important advance.

I've no interest in recounting my whole life's story or even my physics career. Rather, I want to focus on the context of the particular work cited in this year's awards. So I begin this saga with a trip I took with Erick Weinberg, a fellow graduate student, friend, and something of a mentor (he was a year ahead of me) from Cambridge, Massachusetts to Hoboken, New Jersey (I think it was 1970) to a conference to hear our teacher, Sidney Coleman, speak. He was delivering a paper titled "Why Dilatation Generators Don't Generate Dilatations." We had read a written version but hoped that his talk would help us understand it better. It was a several hour drive. Somewhere along the way, I asked Erick to explain to me a bit about what were called Yang-Mills or nonAbelian gauge theories. I had heard the name but was otherwise ignorant. They'd been invented in 1954 and were the last and least understood entry in a short list of what came to be considered the only possible descriptions of fundamental particle interactions. Erick explained the defining basics but told me that nothing was known about their consequences and that many of the most famous senior particle theorists had gotten seriously confused about them. (The list of such notables included Dick Feynman, Shelly Glashow, Abdus Salam, and Steve Weinberg.) And now it seemed that no senior physicist wanted to discuss them; their ignorance and confusion were too embarrassing.

(While delivering my talk live in Stockholm, it occurred to me I should have had a little light or a bell that went off when I mentioned a Nobel laureate-because 
part of my point is to try to understand who is and who isn't. The relevant names are already familiar to the physicist segment of the audience. But for the sake of the general audience, I just raised my finger discretely. Here I'll use a superscript $\mathcal{N}$. So far, there's Yang $^{\mathcal{N}}$, Feynman $^{\mathcal{N}}$, Glashow ${ }^{\mathcal{N}}$, Salam ${ }^{\mathcal{N}}$, Steve Weinberg ${ }^{\mathcal{N}}$, but not Coleman or Erick Weinberg.)

It turns out there was one brave soul, Tini Veltman ${ }^{\mathcal{N}}$, who never gave up on Yang-Mills theory, and, with his best-ever grad student, Gerard 't Hooft ${ }^{\mathcal{N}}$, cracked the case in 1971. I think it worth noting that I, personally, know of no one who claimed to understand the details of 't Hooft's paper. Rather we all learned it from Ben Lee, who combined insights from his own work (that renormalization constants are independent of the choice of ground state in such theories), from hitherto unnoticed work from Russia (Fadde'ev and Popov on quantization and Feynman rules), and from the simple encouragement from 't Hooft's paper that it was possible. (It is amazing how much easier it can be to solve a problem once you are assured that a solution exists!)

The bit of physics I remember best from the Hoboken conference was from a talk by T. D. Lee ${ }^{\mathcal{N}}$. He spoke with confidence that the weak interactions were mediated by a heavy bosonic particle that carried the force, and he gave its mass. (Several years later he was proven right.) The clearest version of that theory had been written down by Steve Weinberg ${ }^{\mathcal{N}}$ in 1967. But no one in that period ever referred to Weinberg's paper. For example, I don't think that Weinberg's paper had any influence on T. D. Lee's thinking. In fact, when what is now known as the Weinberg-Salam model was recognized by the Nobel Foundation, Sidney Coleman published in Science magazine in 1979 a citation search he did documenting that essentially no one paid any attention to Weinberg's Nobel prize winning paper until the work of 't Hooft ${ }^{\mathcal{N}}$ (as explicated by Ben Lee). In 1971 interest in Weinberg's paper exploded. I had a parallel personal experience: I took a one-year course on weak interactions from Shelly Glashow ${ }^{\mathcal{N}}$ in 1970, and he never even mentioned the Weinberg-Salam model or his own contributions to the theory [for which he shared that Nobel prize; by the way, his contribution to that theory was largely his Ph.D. thesis work, done under the direction of Julian Schwinger ${ }^{\mathcal{N}}$, who had already published papers on the non-Abelian gauge bosons as carriers of the weak force in the mid-1960s]. I note again that I also don't personally know anyone who ever read Salam's work on the subject either, except for John Ward, and he was actually the co-author on the relevant papers. He is not a Nobel laureate.

A further aside on the work of 't $\operatorname{Hooft}^{\mathcal{N}}$ and Veltman $^{\mathcal{N}}$, whose contributions were enormously profound and influential, albeit really rather difficult to characterize for a lay audience. One of their many contributions (called, in the business, dimensional regularization) is a tool of essential significance, both for settling issues of principle and for doing explicit calculations. Dimensional regularization also was invented independently for the same purposes and ap- peared in an earlier paper, now mostly forgotten, by Bollini and Giambiaggi.

Coleman's talk in Hoboken was about his then, early understanding of what came to be known as the renormalization group. His thinking was very much influenced by the independent work of Kurt Symanzik and Curt Callan. However, the undisputed champion of the renormalization group was Ken Wilson ${ }^{\mathcal{N}}$ (one of my alltime, absolute heroes), for which he received Nobel recognition. That a prize was given to Wilson $^{\mathcal{N}}$ and Wilson $^{\mathcal{N}}$ alone in 1982 perhaps reflects the depth of his understanding, the precision of his detailed, physical predictions, and his evangelical zeal. We should remember, however, that the renormalization-group work that led to experimentally confirmed predictions, which were in the field of phase transitions and are the substance of the citation for that prize, was all done in collaboration with Michael Fisher; we should remember that the basic, formal work was done independently and published earlier by Wegner and Houghton; and we should remember that the essential physical ideas were articulated independently and earlier by Leo Kadanoff. Furthermore, the renormalization group was actually invented in 1954 by Murray Gell-Mann ${ }^{\mathcal{N}}$ and Francis Low. But even that formulation of the renormalization group appeared in an earlier, independent paper of Stueckelberg and Petermann.

In the early days following the triumph of the Weinberg-Salam model, at one point Glashow $^{\mathcal{N}}$ asked Coleman a practical question that came up in his own work. [The specific technical question was, "What happens if the whole theory has less symmetry than the classical scalar (spin-zero) sector?"] And Coleman answered the question, but he also recognized that the answer was worthy of a deeper, clearer understanding. So, he embarked on its study, in the simplest possible contexts, with my buddy Erick Weinberg. I tagged along in this effort and occasionally made some contribution.

(Here's an anecdote of my first meeting with Niccolo Cabbibo, a charming man, responsible for a monumental contribution to our understanding of the weak interactions and their relation to the strong interactions, which is now largely overlooked because of the telescoping of history into a compact introduction to the present. We were both visiting the University of Chicago, staying at the Windemere Hotel. We chatted over dinner and after as rats scurried between our feet. He is the only person who ever mentioned to me noticing my name in the acknowledgments of Coleman and Weinberg's classic paper.)

During this work with Coleman and Weinberg, one day I wondered and then asked Coleman, "What happens if there are no scalar fields (spin-zero particles) in the first place?" It was an innocent but inordinately profound question which occupied us both quite intensively for the next several months. I learned an enormous amount just working on it. And I benefited from far closer and more extensive interactions with Coleman than he awarded to most of his students-because he was actively working on the problem with me. However, 
I never made what Coleman considered substantial "progress" as measured by his standards. On the other hand, I did many things that, in retrospect, would have been publishable on their own. For example, I was very proud of a trick I invented (only to be told later that it was first done by Heisenberg ${ }^{\mathcal{N}}$ ) for solving [at least in the simplest approximation (what's called $1 / N$ )] what came later to be known as the Gross-Neveu model.

Coleman took a leave of absence from Harvard, taking his sabbatical at Princeton. At that point, I decided I needed a research program on which I could proceed on my own-something that might not meet Coleman's high standards but on which I might have some chance of success. I decided to look into whether the renormalization group had anything to say about the low-energy (or ground-state) behavior of Yang-Mills theory. An analogous analysis for electrodynamics appeared in the classic textbook of Bogoliubov and Shirkov, though Coleman characterized the relevant chapter there as "mysterious." This was a possible approach to the question I articulated regarding no scalar fields, but I thought I might be able to follow the steps of Bogoliubov and Shirkov explicitly as a guide.

A key first step was to know the Yang-Mills beta function. [I assumed (correctly) in my live talk that its definition had been made clear in the earlier remarks of my co-recipients; it is, after all, the minus sign to which I first alluded.] By the way, Erick Weinberg was supposed to compute it for an appendix of his thesis, to carry out a generalization of a renormalization-group flow argument that appears in the Coleman-Weinberg paper, except for a realistic, non-Abelian weak interaction theory. But, in the end, I guess he figured he had enough stuff to get his degree, and it was time for him to move on to something new. I had actually hoped we'd compare notes, but he never attempted the calculation.

I visited Coleman a couple of times in Princeton. When I described to him my new, specific research program, I asked if he knew whether the beta function had already been computed. He thought not but said we should ask David Gross, who was down the hall. David said no, and we discussed briefly then that, while the calculation may have seemed to some to be daunting, it would, in fact, be straightforward.

Fortunately for both of us-and for Frank, too-he was probably wrong, though this episode is fraught with ambiguity. To my knowledge, there are no relevant printed records of the crucial bits of the story, which have been handed down only as folklore, existing in a variety of variant versions.

At a major particle physics meeting in Marseilles the previous year, attended by many particle physics luminaries, Symanzik gave a talk precisely about what came to be known as asymptotic freedom. He described how it could account for the otherwise mysterious results from the Stanford Linear Accelerator (SLAC) on electron-proton scattering. Symanzik knew that the beta functions for other theories were all positive. In fact, many wise people thought there was a general, modelindependent argument for positivity. For example,
Schwinger $^{\mathcal{N}}$ later asked me after hearing me speak on the subject, "What about the positivity of the spectral function?" I.e., intermediate, physical states come with positive probabilities. (This refers to an argument that is, indeed, relevant to other theories.) Symanzik said it would be interesting to know the answer for Yang-Mills theory, and then 't Hooft ${ }^{\mathcal{N}}$ announced it was negative. In some versions of the story, 't Hooft $^{\mathcal{N}}$ spoke up at the question-and-answer period following Symanzik's talk. However, there are attendees of the meeting who have no such recollection. In other versions, it was a private exchange between 't Hooft ${ }^{\mathcal{N}}$ and Symanzik.

There are a variety of first-, second-, and third-hand accounts of why nothing further was heard on this subject from 't Hooft ${ }^{\mathcal{N}}$. I won't repeat them here. ${ }^{1}$ But I'd like to speculate a complementary perspective as to why no one else at the meeting got wind of it or otherwise took any notice. (Admittedly, I wasn't there. So this is pure speculation.) Most theorists' attention then was on weak interactions, and this is a strong interaction issue. But that's not a good enough excuse. People did, after all, talk a lot about the scaling of the Stanford electronproton experiments. Rather, I think Symanzik's speaking style played a crucial role. He was a charming, intense, sweet, and brilliant man. But his live delivery left something to be desired. I remember a different talk I heard him give on a somewhat related subject. He used handwritten slides for an overhead projector (which were the industry standard at the time for technical presentations). However, he obviously wrote out his slides with lined paper underneath as a guide, using every line. So he ended up with over 25 lines of equations and text per page. His handwriting was typical German: undecipherable, at least to Americans, looking like endless updown-up-down-up-down. The clincher, though, was when an equation on one page referred to an equation on another. He'd slap the second slide on top of the first, offset the two by half a line, and point to both.

I slowly and carefully completed a calculation of the Yang-Mills beta function. I happen to be ambidextrous and mildly dyslexic. So I have trouble with left/right, in/out, forward/backward, etc. Hence, I derived each partial result from scratch, paying special attention to signs and conventions. It did not take long to go from dismay over the final minus sign (it was indeed useless for studying low-energy phenomena) to excitement over

\footnotetext{
${ }^{1}$ I will add one conjecture to the list, though it is not something I ever confirmed with 't $\mathrm{Hooft}^{\mathcal{N}}$. It is possible that at that time 't Hooft ${ }^{\mathcal{N}}$ knew the sign of the beta function but not its coefficient. His calculations employed dimensional regularization and dimensional subtraction. From these he would have known the sign of the renormalization constants. However, the fundamental definition of the beta function makes reference to the response of the theory to scale transformations. Dimensional regularization introduces a scale in a subtle way-when one analytically continues away from the superficially scale invariant dimensions. How the traditional renormalization group is represented in this context is something that was worked out only a couple of years later.
} 
the possibilities. I phoned Sidney Coleman. He listened patiently and said it was interesting. But, according to Coleman, I had apparently made an error because David Gross and his student had completed the same calculation, and they found it was plus. Coleman seemed to have more faith in the reliability of a team of two, which included a seasoned theorist, than in a single, young student. I said I'd check it yet once more. I called again about a week later to say I could find nothing wrong with my first calculation. Coleman said yes, he knew because the Princeton team had found a mistake, corrected it, and already submitted a paper to Physical Review Letters.

On learning of the Gross-Wilczek-Politzer result, Ken Wilson $^{\mathcal{N}}$, who might have thought of its impossibility along the same lines as I attributed to Schwinger ${ }^{\mathcal{N}}$, above, knew who to call to check the result. He realized that there were actually several people around the world who had done the calculation, en passant as it were, as part of their work on radiative corrections to weak interactions in the newly popular Weinberg-Salam model. They just never thought to focus particularly on this aspect. But they could quickly confirm for Wilson $^{\mathcal{N}}$ by looking in their notebooks that the claimed result was, indeed, correct.

Steve Weinberg ${ }^{\mathcal{N}}$ and Murray Gell-Mann ${ }^{\mathcal{N}}$ were among those to instantly embrace non-Abelian color $\mathrm{SU}(3)$ gauge theory as the theory of the strong interactions. In Gell-Mann's case it was in no small part because he had already invented it (!) with Harald Fritzsch and christened it QCD. He had previously articulated three solid arguments for choosing this particular theory. (For the physicists, those arguments were baryon statistics, $\pi \rightarrow 2 \gamma$, and the electron-positron annihilation cross section.) And asymptotic freedom, i.e., the negative beta function, was the clincher. I'd only heard of Gell-Mann $^{\mathcal{N}}$ and Fritzsch's work second hand, from Shelly Glashow ${ }^{\mathcal{N}}$, and he seemed think it shouldn't be taken too seriously. I only later realized it was more Glashow's mode of communication than his serious assessment of the plausibility of the proposal. In any case, I had completely lost track of Gell-Mann ${ }^{\mathcal{N}}$ and Fritzsch's QCD.

After the first seminar I ever gave on this subject (it was at MIT), I was approached by Ken Johnson (who himself had done pioneering work on the renormalization group years earlier) and Vicki Weisskopf. "Very nice," they said. "Too bad that it is in glaring contradiction to at least two important classes of experiments." One problem was the electron-positron cross section, which had only gotten much worse since Gell-Mann ${ }^{\mathcal{N}}$ and Fritzsch's proposal of QCD, and the other was the issue of large-angle products in proton-proton collisions. There were many more energetic particles produced than expected (naively) from QCD. By the way, this second issue attracted Dick Feynman's attention. And it wasn't until a couple of years later and his careful analysis with Rick Field that QCD was reconciled with those experiments. Only then did Feynman ${ }^{\mathcal{N}}$ join the ranks of the believers.
The experimentally measured electron-positron cross section (as a function of increasing collision energy) had leveled off-instead of continuing to drop steeply, which was thought to be a QCD prediction. In Aspen, Colorado, in the summer of 1974, I crossed paths with Ken Wilson ${ }^{\mathcal{N}}$, who, characteristically succinct, said, "It's charm, and it's not short distance." Tom Appelquist and I made it our task to understand those oracular comments and flesh out their consequences. By the end of the summer, the reconciliation of QCD with the experimental measurements was pretty clear to us. Tom toured the country explaining our work. His seminars included a sketch of what the cross section really was as opposed to what the experimentalists reported and an estimate, albeit technically an upper bound, on the astoundingly long lifetime of a particle that was being produced and decaying as yet unnoticed. Many people heard those talks and remember them, and there is at least one, objective written record of their existence: Sid Drell gave an account in a piece he wrote subsequently for Scientific American about charm. At the time, there were already many what-proved-to-be-wrong papers trying to interpret the electron-positron experiments, and the SLAC experiment leader Burt Richter ${ }^{\mathcal{N}}$ was touring the country explaining that he had made the monumental discovery that the electron was actually a little hadron, i.e., a strongly interacting particle like the proton, only much smaller in diameter. (This discovery, or at least the same experimental results, had been observed a few years earlier at the Cambridge Electron Accelerator, a joint Harvard-MIT venture. But no one believed it, and the machine was decommissioned.) Appelquist and I were drafting a paper. But I was the conservative one, perhaps overly influenced, I later realized, by a talk that I had heard by Steve Adler as to how large the discrepancy between naive QCD calculation and experimental measurements could be before the theory was in definite trouble. I focused on the things we could most reliably compute and did not appreciate the correctness of Tom's more general arguments.

In November that Fall came the experimental announcements. SLAC observed a particle (they called it the $\psi$ ) and ultimately observed a whole cross section just as predicted by Appelquist. And observation was simultaneously announced by Sam Ting $\mathcal{N}$, in an experiment that identified a pimple, which $\operatorname{Ting}^{\mathcal{N}}$ eponymously titled the $\mathcal{J},{ }^{2}$ on what had been known as Lederman's shoulder. (That's Leon Lederman ${ }^{\mathcal{N}}$.) That is to say that Ting's experiment had actually been done earlier by Lederman $^{\mathcal{N}}$. The earlier experiment had cruder resolution, but it clearly indicated that there was something anomalous at just that energy.

Appelquist and I hurriedly dashed off a short version of our work to Physical Review Letters, where it was immediately and unequivocally rejected by senior editor Sy Pasternak. It was against that journal's policy to let

\footnotetext{
${ }^{2} \mathrm{~A}$ reasonable approximation to the relevant Chinese character.
} 
authors engage in the coining of frivolous, new terminology. In the case at hand, our friend and colleague Alvaro De Rújula, on hearing of our work, had coined the term "charmonium," which in a single word was able to transmit the central new idea of the paper to any serious particle physics reader. Ultimately, Shelly Glashow ${ }^{\mathcal{N}}$ brokered a compromise with Pasternak. We could use "charmonium" in the text but not in the title. The negotiations caused a delay of a couple of weeks-a long time in those heady days. As a consequence, publication came alongside several other long-since-forgotten papers, instead of being hard on the heals of the experimental discovery.

That our explanation was correct was soon widely appreciated, and it convinced almost all of the remaining skeptics of the validity of QCD. I suspect that the consensus on this issue was a major contributing factor to the Swedish Royal Academy's recognition within just a couple of years of Richter ${ }^{\mathcal{N}}$ and Ting's discovery.

I hope you all now understand why I owe Tom Appelquist a huge, profound, and public apology. We certainly could have submitted for publication in September substantially the same paper we ultimately wrote two months later.

Now, somewhat out of chronological order, I'd like to express my thanks to my old friend and collaborator Howard Georgi. After the calculation of the beta function, it was fairly obvious what should be done next. One had to re-do some calculations that had been done earlier by Norman Christ, Brosl Hasslacher, and Al Mueller but in the context of what was now, obviously, the right theory. Here, again, a missing name from that collaboration but who had a major impact was Georgio Parisi. Well, Howard Georgi checked up periodically on my progress, and I admitted having some technical trouble. So he volunteered to help, and we went on to do an enormous number of clever things together.

Apropos clever, there are some advances that require considerable mental struggle and lengthy argumentation, only to virtually disappear as nonissues, because they're simply obvious from a newer perspective. For example, the fact that quarks could have a mass, something unambiguously quantifiable and measured in grams-in spite of their never existing as isolated particles-was one such issue on which I battled with many physicists, including, for example, Gell-Mann ${ }^{\mathcal{N}}$ and Steve Weinberg ${ }^{\mathcal{N}}$. The heavy charm quark gave impetus to those considerations, but there was a conceptual battle that had to be fought against older prejudices formed in the limited context of the "light" quarks. Younger physicists today can't even imagine that there was ever an issue.

Heavy quarks appeared once again in my research life. Joe Polchinski asked Mark Wise, a colleague of mine at Caltech, a question about heavy quark calculations, which Mark and I proceeded to answer. It was again a case where, unbeknownst to us, the work had already been done, this time by Misha Shiffman and Mike Voloshin in the Soviet Union. Furthermore, I again missed the most important phenomenological con- sequences of that line of thought. Those had to wait for the collaboration of Mark Wise with Nathan Isgur. That heavy quark physics depends only trivially on the actual value of the heavy quark mass was obvious to me and probably most anyone else who gave it a thought. What Isgur and Wise noted was that in a world with more than one type of heavy quark, this gives rise to symmetries of monumentally useful importance. (The second heavy quark, the so-called bottom quark, was identified only several years after the first, i.e., the charmed quark.)

The establishment by the mid-1970s of QCD as the correct theory of the strong interactions completed what is now known prosaically as the Standard Model. It offers a description of all known fundamental physics except for gravity, and gravity is something that has no discernible effect when particles are studied a few at a time. However, the situation is a bit like the way that the Navier-Stokes equation accounts for the flow of water. The equations are at some level obviously correct, but there are only a few, limited circumstances in which their consequences can be worked out in any detail. Nevertheless, many leading physicists were inclined to conclude in the late 1970s that the task of basic physics was nearly complete, and we'd soon be out of jobs. A famous example was the inaugural lecture of Stephen Hawking as Lucasian Professor of Mathematics, a chair first held by Isaac Barrow at Cambridge University. Hawking titled his lecture, "Is the End in Sight for Theoretical Physics?" And he argued strongly for "Yes."

But more recent observations of astronomers have turned things on their heads. Recall, if you will, that among the many stupendous insights of Isaac Newton, the second Lucasian Professor, was the idea that the stuff of the heavens was the same stuff as matter here on Earth. This was revolutionary. And he asserted that the laws that governed the motion of stuff in the heavens were the same laws as applied to matter on Earth. (That there are laws at all may be his most profound insight. It is certainly what came to define the whole discipline of physics.) For three centuries we accumulated stunning detailed confirmation of these of Newton's assertions. But in a very fundamental way both of these ideas now appear to be about as wrong as they possibly could be-at least that's the simplest interpretation of our current large-scale astrophysical observations. It turns out that we haven't a clue what virtually all of the matter in the Universe consists of-except that it's not made of the particles that make up matter on Earth or in the stars. Furthermore, the force which governs the largest scale motions in the Universe has nothing to do with the forces of the Standard Model or with gravity as it is familiar here on Earth.

There is a very active field of theoretical research which seeks to go beyond the Standard Model. Success in these endeavors would mean explaining the apparently arbitrary aspects of the Standard Model; success would mean bringing an account of gravity into the picture; and success would mean illuminating the previously mentioned issues in astrophysics. However, we now face a very serious problem in advancing the ex- 
perimental frontier, a problem which few people like to discuss. It seems to me that ever since Leeuwenhoek, advances in the resolving power of our "microscopes" have come with similar investments of capital and manpower. I.e., an increase by an order of magnitude in the one required an increase by roughly an order of magnitude in the other-at least once we average over fits and starts and brilliant insights. The last big machine planned and canceled in the U.S. was to cost about $\$ 10$ billion. (That's $\$ 10^{10}$.) That would have allowed us to reach distances small enough to study the interactions of weak bosons directly. The realm of the conjectured "unification" of the forces of the Standard Model, the realm of their possible unification with gravity, and the basic physics of string theory, the most widely pursued approach to a physics more fundamental than the Standard Model, are all more than a dozen orders of magnitude further away. However, $\$ 10^{22}$ is simply not available for this line of research (or anything else for that matter).

The question of the benefit of this work incurred on mankind, an aspect stipulated in Mr. Nobel's will, is a whole other topic. But, as I said at the outset, I certainly appreciate the care and wisdom invested by the Royal Academy in identifying noteworthy advances in fundamental physics-and in identifying the particular advance that we celebrate today. The reality of the actual progress of science is, however, often very complicated, as I hope I have conveyed from my few examples. The committees of the Academy know this full well, but their deliberations are confidential. I felt strongly that more of the public should contemplate these matters if they wish to understand not just the ideas of science but also how they have developed. I also hope that more of the scientific community would remember them, too.

My presentation in Stockholm ended at this point, but, in the days that followed, it prompted a variety of comments, questions, and exchanges. I'd like to add here a brief version of one of them. I was asked, point blank, what I actually thought of the 2004 Nobel Prizes in Physics, aside from the obvious personal considerations. And this is a distilled version of my reply. Recognition of the theory of the strong interactions is an obvious choice-for all the reasons that have been discussed in my co-recipients' lectures, in the presentation speech in Stockholm by Lars Brink, in the assembled material of the Nobel Foundation, and in the wide coverage elsewhere. However, in my view, getting to our current level of understanding has been a rich and complex story. Nevertheless, I believe that it is the overwhelming consensus (but by no means unanimous) opinion of researchers in the field - and I personally agreethat the discovery of asymptotic freedom was a genuinely crucial event. For some, it made everything clear. For others, it was only the beginning. And for yet others, it was the beginning of the final chapter. But in any case, it was key.

The two books I would recommend first which give excellent accounts of this epoch in particle physics (and more) are The Second Creation, by Robert Crease and Charles Mann and Constructing Quarks, by Andrew Pickering. The first is colorful in its rendition of the personalities, rather accurate in its physics, and totally accessible to the interested layman. The second is a more scholarly endeavor. Pickering began his career as a particle theorist, a contemporary of mine. He includes considerable scientific detail but still aims at a nontechnical audience. Two marvelous books on twentieth century physics for the interested layman which focus more on scientific substance rather than historical process are both by Heinz Pagels, something of a self-styled New York City dandy, but as charming a person as could be. He died young but just as he dreamed it would be. The Cosmic Code is about quantum mechanics, and Perfect Symmetry covers more of the sweep of particle physics and cosmology.

I have not sought out the actual published references for the relevant points in my narrative. They're not hard to find, but Les Prix Nobel is not a refereed journal. And yet, there is a potentially enlightening aspect to my having put this together purely from memory in October and November of 2004. While standard references are unequivocally available in the published record, what actually transpired, leading to those publications, is not. We rely on people's personal accounts. And now we enter the interesting realm where participants in the same event may have very different and mutually contradictory perceptions of what transpired, and those perceptions may shift as time passes. While intentional deception is not an unheard of phenomenon, these phenomena affect the reports of people with the highest integrity. Although evaluating the accuracy of my personal recollections may be very difficult, at least it would be possible to see how good my memory is with respect to items that can be confirmed or refuted. 